\title{
PERFIL DE PREMATUROS NASCIDOS VIVOS EM UM MUNICÍPIO DO INTERIOR DA BAHIA
}

\author{
$\underline{\text { Taynara Bispo Conceição }}^{1}$; Luciano Marques dos Santos $^{2}$ \\ 1. Voluntário PEVIC/CNPq, Graduando em Enfermagem, Universidade Estadual de Feira de Santana, e-mail: \\ taynara_tatay@hotmail.com \\ 2. Orientador, Departamento de saúde, Universidade Estadual de Feira de Santana, e-mail: \\ lucmarxenfo@yahoo.com.br
}

\section{PALAVRAS-CHAVE: Enfermagem Obstétrica. Trabalho de Parto prematuro. Recém-nascido prematuro.}

\section{INTRODUÇÃO}

A prematuridade é resultante de ocasiões diversas e imprevisíveis, em todos os lugares e classes sociais. Representa também um grande problema de Saúde Pública, pois acarreta às famílias e à sociedade em geral um custo social e financeiro de difícil mensuração, além de ser um determinante de morbimortalidade neonatal, principalmente em países em desenvolvimento. Segundo relatório da Organização Mundial da Saúde (OMS), a incidência dos partos prematuros é maior nos países pobres e atualmente está em torno de 12\% maior do que em nações mais desenvolvidas (9\%)(Ramos; Cuman, 2009; Howson;Kinney;Lawn, 2012).

O relatório "Born too Soon", divulgado pela OMS em 2012, mostrou que 15 milhões de bebês nascem antes do tempo por ano no mundo. O Brasil e os Estados Unidos estão entre os dez países com os maiores números de partos prematuros (Howson; Kinney; Lawn, 2012). O Brasil aparece em décimo lugar, com cerca de três milhões de crianças ao ano, das quais 279 mil são prematuros, sendo 35.000 com peso ao nascer inferior a $1.500 \mathrm{~g}$. A taxa brasileira é 9,2\% dos bebês prematuros, igual à da Alemanha e inferior à dos Estados Unidos, que chega a 12\% (Brasil, 2018).

Baseado nos dados do Sistema de Informações sobre Nascidos Vivos (SINASC), foi realizado um estudo no estado de Santa Catarina, em 2005, onde mostrou que o peso médio das crianças ao nascer foi de $3.232,8 \mathrm{~g}$ variando de $500 \mathrm{~g}$ a $6.035 \mathrm{~g}$. As características dos prematuros foram: sexo masculino com prevalência de $6,2 \%$; nascidos de mães com idade superior a 40 anos $(8,8 \%)$ e nascidos de partos por cesariana (6,6\%) (Cascaes et al., 2008).

Considerando a estatística de prematuros nascidos vivos, sabe-se que um percentual considerável dos neonatos pré-termo precisa de auxílio para iniciar a transição cardiorrespiratória para a adaptação à vida extrauterina. Dados da Rede Brasileira de Pesquisas Neonatais indicam que, em 2010, dos nascidos vivos prematuros de extremo baixo peso e sem malformações, $60 \%$ precisaram de ventilação com pressão positiva, dos quais $40 \%$ melhoraram com a ventilação por máscara facial e que aproximadamente $6 \%$ foram ventilados com cânula traqueal e receberam massagem cardíaca e/ou medicações na sala de parto (Brasil, 2012).

Assim, este plano de trabalho se justifica em gerar conhecimento das características de um grupo populacional contribuindo para a redução dos índices dos indicadores de saúde, principalmente o coeficiente de mortalidade infantil. Esses dados alicerçarão, 
direcionarão e subsidiarão as ações propostas pelos diversos a assistência à saúde, bem como sua forma de execução. Portanto, uma avaliação contínua desses indicadores de saúde pelas administrações públicas forneceria estratégias para assistência integral à criança e instrumentos para redução da incidência de morbimortalidade infantil.

Diante disso, questiona-se: Qual o perfil de prematuros nascidos vivos em um município no interior da Bahia? Cujo o objetivo é identificar o perfil de recém-nascidos prematuros nascidos vivos em uma cidade do interior da Bahia.

\section{MATERIAL E MÉTODOS OU METODOLOGIA (ou equivalente)}

Trata-se de um estudo do tipo descritivo, exploratório e quatitativo de caso controle, vinculado à pesquisa multicêntrica intitulada "Parto prematuro: estudo dos fatores associados para construção de estratégias de prevenção", que foirealizada em quatro municípios brasileiros sendo dois no nordeste e dois na região sul. $O$ estudo foi realizado no município de Feira de Santana, Bahia, no Hospital Inácia Pinto dos Santos (HIPS), e no Hospital Geral Cleriston Andrade (HGCA).

A amostra do estudo multicêntrico foi estimada em 600 entrevistadas, divididas em 300 casos e 300 casos controles, tomando como base dados do município do Rio Grande, e pelo fato do Rio Grande do Sul ter o maior índice de prematuridade entre os estados brasileiros. Este cálculo foi realizado esperando uma prevalência de partos prematuros em torno de $12 \%$, com o máximo de $10 \%$ de perdas. Para este estudo, foram utilizados os dados dos 333 casos, em particular, dos recém-nascidos prematuros. Portanto, não foram adotados critérios de inclusão e de exclusão.

Os dados foram coletados desde abril de 2014, com finalização em fevereiro de 2016. Para tanto, foi utilizadoum formulário contendo informações relacionadas às condições sociodemográficas, reprodutivos, gestacionais, hábitos de vida e do recém-nascido prematuro. Foram consideras como variáveis deste estudo:Sexo; Idade gestacional; Peso do nascimento; Apgar do primeiro minuto de vida; Apgar quinto minuto de vida; Necessidade de reanimação; Medidas utilizadas durante a reanimação; Necessidade de internamento; Local do internamento e Motivos da internação.

Os dados foram tabulados em planilhas eletrônicas e analisados através do programa StatisticalPackage for Social Sciences (SPSS), versão 15.0. Foram calculadas as frequências absolutas e relativas das variáveis.

Antes da coleta de dados, as puérperas foram informadas quanto aos riscos, benefícios, objetivos da pesquisa, quanto ao caráter voluntário de sua participação e da preservação do seu anonimato, mediante leitura e assinatura do Termo de Consentimento Livre e Esclarecido, o qual assegurou os princípios éticos da pesquisa envolvendo seres humanos. Este estudo respeita a Resolução 466/2012 do Conselho Nacional de Saúde sendo devidamente aprovado pelo Comitê de Ética na Pesquisa (CEP) da Universidade Federal do Rio Grande através do parecer de número 134/2013 e no CEP da UEFS através do parecer 643.668.

\section{RESULTADOS E/OU DISCUSSÃO}


A análise dos dados quantitativos permitiu traçar o perfil dos recém-nascidos prematuros nascidos vivos e a necessidade dos mesmos da reanimação em sala de parto e a necessidade de cuidados intensivos ou semi-intensivos.

PERFIL DE PREMATUROS NASCIDOS VIVOS EM UM MUNICÍPIO DO INTERIOR DA BAHIA.

De um total de 653 recém-nascidos internados no período estudado, 51,1\% casos de nascidos vivos prematuros. De acordo com as variáveis relacionadas ao recém-nascido prematuro, 50,6\% foram do sexo masculino, 55,3\% tiveram baixo peso ao nascer (média de $2.091 \mathrm{~kg}$ ), 77,7\% Apgar maior do que 7 no primeiro minuto de vida (média de 7) e este mesmo valor do boletim no quinto minuto de vida em 93,0\% dos casos investigados (média de 8) (Tabela 1).

Com relação a idade gestacional com base pela DUM 47,2 (média de 34,3 semanas) foram classificados com moderado, e com base pela ecografia 46,9\% (média de 33,7 semanas) e pelo método de Capurro 50,7\% (média de 33,6 semanas) foram classificados com limítrofe respectivamente. (Tabela 1).

Tabela 1 -Características de prematuros nascidos vivos em dois hospitais públicos da cidade de Feira de Santana-Bahia de abril 2014 a novembro de 2015.

\begin{tabular}{|c|c|}
\hline Variáveis & $\mathbf{N}(\%)$ \\
\hline \multicolumn{2}{|l|}{$\operatorname{Sexo}(N=333)$} \\
\hline Masculino & $168(50,6 \%)$ \\
\hline Feminino & $164(49,4 \%)$ \\
\hline \multicolumn{2}{|c|}{ Peso ao Nascer $(\mathrm{N}=331 *)$} \\
\hline Baixo Peso Extremo & $19(5,7 \%)$ \\
\hline Muito Baixo Peso & $46(13,9 \%)$ \\
\hline Baixo Peso ao nascer & $183(55,3 \%)$ \\
\hline Peso Normal & $83(25,1 \%)$ \\
\hline \multicolumn{2}{|l|}{ Apgar $1(\mathrm{~N}=301 *)$} \\
\hline$<7$ & $67(22,3 \%)$ \\
\hline$\geq 7$ & $234(77,7 \%)$ \\
\hline \multicolumn{2}{|l|}{ Apgar 5 (N=301*) } \\
\hline$<7$ & $21(7,0 \%)$ \\
\hline$\geq 7$ & $280(93,0 \%)$ \\
\hline \multicolumn{2}{|l|}{$\overline{\mathrm{DU}} \mathbf{\mathrm { N }}(\mathbf{N}=\mathbf{2 1 4} *)$} \\
\hline Prematuro Extremo & $21(9,8 \%)$ \\
\hline Moderado & $92(43,0 \%)$ \\
\hline Limítrofe & $101(47,2 \%)$ \\
\hline \multicolumn{2}{|l|}{ Ecografia $\left(N=318^{*}\right)$} \\
\hline Prematuro Extremo & $32(10,1 \%)$ \\
\hline Moderado & $149(46,9 \%)$ \\
\hline Limítrofe & $137(43,0 \%)$ \\
\hline \multicolumn{2}{|c|}{ Método de Capurro (N=229*) } \\
\hline Prematuro Extremo & $25(10,9 \%)$ \\
\hline Moderado & $116(50,7 \%)$ \\
\hline Limítrofe & $88(38,4 \%)$ \\
\hline
\end{tabular}

Fonte: Coleta de dados. 
* As diferenças no total da amostra são decorrentes de dados ignorados que não foram encontrados no prontuário do recém-nascido ou materno, ou a entrevistada não soube/quis informar, ou não se aplicam a questão.

\section{ASSISTENNCIA IMEDIATA EM NASCIDOS VIVOS EM UM MUNICÍPIO DO INTERIOR DA BAHIA.}

No que se refere a assistência imediata no nascimento $60,5 \%$ dos recém-nascidos foram reanimados, sendo que $21 \%$ necessitaram de $\mathrm{O}_{2}$ inalatório, $42,3 \%$ foram encaminhados para unidade de alojamento conjunto. Entretanto, 32,2\% foram hospitalizados em unidade semi-intensiva neonatal e $25,5 \%$ em cuidados intensivos, sendo a disfunção respiratória o principal motivo de hospitalização nestas unidades neonatais $(41,2 \%)$ (Tabela 2).

Tabela 2 - Perfil de prematuros nascidos vivos em dois hospitais públicos da cidade de Feira de Santana-Bahia de abril 2014 a novembro de 2015.

\begin{tabular}{ll}
\hline \hline \multicolumn{1}{l}{ Variáveis } & \multicolumn{1}{c}{$\mathbf{N}(\%)$} \\
\hline $\begin{array}{l}\text { Necessidade de Reanimação em sala de parto } \\
(\mathbf{N}=332 *)\end{array}$ & $201(60,5 \%)$ \\
Sim & $131(39,5 \%)$ \\
Não & \\
Que atendimento necessitou em sala de parto \\
$(\mathbf{N = 1 7 5 * )}$ & $70(21,0 \%)$ \\
$\mathrm{O}^{2}$ inalatório & $25(7,5 \%)$ \\
VPP por máscara & $20(6,0 \%)$ \\
VPP por TET & $4(1,2 \%)$ \\
Massagem cardíaca externa & $4(1,2 \%)$ \\
Drogas & $52(15,7 \%)$ \\
Outro & \\
Local de internação (N=333) & $141(42,3 \%)$ \\
Alojamento conjunto & $107(32,2 \%)$ \\
Semi-intensiva neonatal & $85(25,5 \%)$ \\
UTI Neonatal & \\
Motivo principal da internação & \\
(N=195*) & $137(41,2 \%)$ \\
Disfunção respiratória & $58(17,6 \%)$ \\
Outros &
\end{tabular}

Fonte: Coleta de dados.

* As diferenças no total da amostra são decorrentes de dados ignorados que não foram encontrados no prontuário do recém-nascido ou materno, ou a entrevistada não soube/quis informar, ou não se aplicam a questão.

\section{DISCUSSÃO}

Quanto às características dos prematuros nascidos vivos (Tabela 1), este estudo mostrou predomínio de nascimentos de prematuros do sexo masculino, apesar de que a questão de gênero não é significativa. A maioria dos estudos presentes na literatura apresenta maior registro de nascimentos pré-termo no sexo masculino (Piccoli et al., 2012; Drummond et al., 2014; Silva; Pelloso, 2009). Estudos realizados na Holanda demonstraram que o sexo masculino para o recém-nascido tinha associação com 
aumento do risco de sofrimento fetal, enquanto foi observado efeito protetor do sexo feminino (Cunha et al., 2004).

Quanto ao peso ao nascer constitui um dos pilares das variáveis de requisito para a sobrevivência infantil que, quando inferior a $2.500 \mathrm{~g}$ é considerado um dos principais determinantes da mortalidade neonatal e da morbidade infantil (Rego et al., 2010). O peso do recém-nascido é estimado como fator decisivo para a análise do desenvolvimento pós-natal (Salge et al., 2009). Esse estudo demonstrou que $25.1 \%$ dos nascidos vivos prematuros apresentaram peso normal ao nascimento e $74.9 \%$ apresentaram baixo peso ao nascer (Tabela 1), ou seja, tiveram peso menor que $2.500 \mathrm{~g}$, o que identificam uma população de recém-nascidos com condições de saúde desfavoráveis, associadas às principais causas de morbimortalidade neonatal e infantil (Rego et al., 2010).

A organização Mundial da Saúde (OMS) define como Baixo peso ao nascer o recémnascido com peso menor que $2.500 \mathrm{~g}$, independentemente da idade gestacional. O Baixo peso ao nascer é considerado como um fator definitivo que leva a desnutrição, além de representar as condições nutricionais do recém-nascido, da gestante, a influencia no crescimento e desenvolvimento da criança e, com o tempo, reflete na qualidade da saúde do adulto (Ramos; Cuman, 2009; Motta et al., 2005).

Um estudo realizado no Hospital das Clínicas da Universidade Federal de Goiás, que é referência regional para o atendimento de gestação de alto risco, mostrou que a média de pesos ao nascer foi de $2350 \mathrm{~g}$, caracterizando baixo peso ao nascer (Salge et al., 2009). No Rio Grande do Sul no município de Porto Alegre, em 2012 um estudo do tipo caso-controle feito pela base de dados Sistema de Informações sobre Nascidos Vivos (SINASC), o peso ao nascer menor que $2500 \mathrm{~g}$ apresentou quatro vezes mais chances de estar associado ao nascimento prematuro (Oliveira et al., 2016).

Com base na literatura, torna-se grande a preocupação para profissionais de saúde o baixo peso ao nascer, pois se associa à morbimortalidade neonatal e infantil. Os recémnascidos com peso menor que $2.500 \mathrm{~g}$, podem ser justificadas de duas condições opostas, a prematuridade ou restrição do crescimento intrauterino, que podem ser causadas por diversos fatores inclusive redução da idade gestacional, esses fatores do baixo peso ao nascer, podem atuar de forma isolada ou sinérgica e em graus instáveis (Ramos; Cuman, 2009).

Em Santa Maria foi realizado um estudo do tipo transversal, que evidenciou $78 \%$ dos bebês com baixo peso nasceram prematuros, apontando a prematuridade como a principal responsável pelo baixo peso ao nascer (Rades; Bittar; Zubaig, 2004). A relação entre a idade gestacional e o peso ao nascer é direta, ou seja, quanto menor o tempo de desenvolvimento dentro do útero, menores são os tamanhos e os pesos dos recémnascidos (Oliveira et al., 2016).

O índice de prematuridade desse estudo foi definido através idade gestacional, foi considerado essa variável em menos de 37 semanas para caracterizar os nascimentos prematuro, sendo analisada através da última data de menstruação (DUM), ecografia e método de capurro (Tabela 1).

Os prematuros ainda são classificados conforme grau de prematuridade: os limítrofes são os que nascem com idade gestacional entre 35 e 36 semanas; os prematuros 
moderados com idade gestacional entre 31 e 34 semanas; e os prematuros extremos com idade gestacional inferior ou igual a 30 semanas (WHO, 2002).

Uma avaliação precisa da idade gestacional é primordial para tomar decisões sobre cuidados obstétricos que afetem o recém-nascido prematuro. Para obter a idade gestacional precisa e confiável em alguns casos, torna-se difícil devido a fatores, como a incerteza quanto à data da última menstruação e o início tardio do pré-natal, que alteram a idade gestacional baseada no cálculo pelo ultrassom (Mwamakamba; Zucchi, 2014). Isso dificultou a coleta de dados devido à falta dessas informações gerando números ignorados em nosso banco.

No Brasil, nascem cerca de três milhões de crianças ao ano, das quais 200.000 apresentam idade gestacional abaixo de 37 semanas, sendo $35.000 \mathrm{com}$ peso ao nascer inferior a 1.500g (Branco; Guinsburg, 2013). No estudo feito em Guarapuava, as condições da gestação e parto e o tempo da gestação, considerando-se prematuridade extrema, ou seja, tempo de gestação menor que 31 semanas, foi observada uma frequência de 26\% para os prematuros nascidos vivos (Ramos; Cuman, 2009).

Neste estudo, uma das classificações da idade gestacional foi determinada com base no método de capurro, que atualmente não é o mais indicado, pois é avalia mais as características neurológicas do que as corpóreas somáticas. O ideal seria o escore de New Ballard, que consiste em exame físico e neurológico feito por neonatologistas nas primeiras 12 horas de vida. Esse teste foi considerado por haver um padrão previsível de mudanças ocorridas durante a gestação e por ser um teste preciso, quando comparado ao de capurro e outros (Mwamakamba; Zucchi, 2014).

Outra variável importante para avaliar no prematuro, é o índice de APGAR que é uma escala de risco que representa as condições clínicas do recém-nascido, de forma que caracterize e pontue de acordo com a presença da respiração, cor da pele, batimentos cardíacos, tônus musculares e resposta a estímulos (Oliveira; Moura; Silva, 2007). Esta escala foi criada na década de 1950, e sugeria que se o escore de um concepto permanecesse baixo aos cinco minutos de vida, existiria grande probabilidade de problemas neurológicos (Cremonese et al., 2012).

O índice de Apgar baixo é útil para identificar os recém-nascidos que necessitam de cuidados adicionais. Um índice de Apgar com nota maior que 7 significa que o bebê é saudável e sugere que não terá problemas futuros. Quando a nota for menor que 7 é sinal de alerta com alterações fisiopatológicas e maturidade do recém-nascido (Ramos; Cuman, 2009).

No que se refere ao índice de Apgar no primeiro minuto de vida, a pesquisa mostra que $22,3 \%$ nota insatisfatória (menor que 7), e apenas $7 \%$ obteve nota insatisfatória no quinto minuto de vida. Esse dado é interessante, pois mostra que, entre todos os prematuros, um percentual de 7\% manteve o índice de Apgar insatisfatório. Isso revela que mesmo que tenham nascido com dificuldades, houve recuperação rápida na avaliação após 5 minutos, também indica um bom prognóstico e grandes chances de sobrevida. Também podemos dizer que $7 \%$ dos nascidos vivos tiveram a necessidade de atendimento de maior nível de complexidade e possíveis chances de complicações e sequelas. A classificação de um índice de Apgar inferior a 7 é indicativa de 
precariedade nas funções vitais do recém-nascido, havendo necessidade de intervenção imediata da equipe de saúde (Cremonese et al., 2012).

Um estudo feito em Belo Horizonte, registrou 85,9\% e 97,9\% de recém-nascidos que receberem nota maior que 7 no primeiro e quinto minuto respectivamente (Rego et al., 2010). Os recém-nascidos prematuros em Guarapuava, $79 \%$ receberam nota do índice de Apgar maior que 7 (Ramos; Cuman, 2009).

Percebe-se, portanto, que além desse estudo, outros estudos realizados em municípios do Brasil, tem conseguido melhorar as condições de nascimento, e comprovam a relação de melhores condições maternas e fetais durante o pré-natal, na gestação e qualidade na assistência ao parto.

Com relação a necessidade de reanimação em prematuros, segundo a literatura observase uma importante influência da idade gestacional e do peso ao nascer em relação as condições dos recém-nascidos e na necessidade de reanimação neonatal. A necessidade de procedimentos de reanimação e índice de mortalidade é maior, quanto menor a idade gestacional e/ou peso ao nascer (Drummond et al., 2014).

Geralmente, 2 em cada 3 recém-nascidos com peso de nascimento menor que $1.500 \mathrm{~g}$ precisam de reanimação. Um em cada 10 recém-nascidos necessita de ventilação com pressão positiva para iniciar e/ou manter movimentos respiratórios efetivos; um em cada 100 neonatos precisa de intubação e/ou massagem cardíaca; e um em cada 1.000 requer intubação, massagem e medicações, desde que a ventilação seja aplicada adequadamente (Branco; Guinsburg, 2013).

$\mathrm{O}$ ato de reanimar um recém-nascido, principalmente os prematuros, não é livre de riscos. Há a possibilidade de complicações graves, tais como pneumotórax, hemotórax, fratura de costelas, entre outras, além de poder contribuir, de forma direta ou indireta, para o aumento na incidência de patologias tardias (Drummond et al., 2014).

Sabe-se que um percentual considerável dos prematuros precisou de auxílio para iniciar a transição cardiorrespiratória necessária para adaptar-se à vida extrauterina. Estima-se que, no país a cada ano, 300.000 crianças necessitem ajuda para iniciar e manter a respiração ao nascer e cerca de 25.000 prematuros de baixo peso precisem de assistência ventilatória na sala de parto (Branco; Guinsburg, 2013).

Segundo um documento científico do programa de reanimação neonatal da sociedade brasileira de pediatria, por ano no Brasil, cerca de 25 mil recém-nascidos de baixo peso extremo necessitam de assistência ventilatória adequada na sala de parto, sendo esta considerada como ponto crítico para o sucesso da reanimação neonatal, fazendo com que os pulmões do recém-nascido se inflem e, com isso haja dilatação da vasculatura pulmonar e hematose apropriada. (Branco; Guinsburg, 2013).

Um estudo publicado pela Rede Americana de Pesquisas, 9.565 neonatos prematuros, mostra que $67 \%$ deles receberam ventilação com pressão positiva ao nascer por meio de cânula traqueal, $8 \%$ necessitaram de massagem cardíaca e 5\% de medicações (Stoll et al., 2010). Enquanto nesse estudo, 60,5\% necessitaram de reanimação em sala de parto. Dentre as formas de assistência, $21 \%$ necessitaram da inalação de $\mathrm{O}^{2}$ inalatório, além da necessidade da ventilação com pressão positiva por máscara, ventilação com pressão positiva por meio de cânula traqueal, massagem cardíaca externa e medicações. 
A elevada necessidade de ajuda para iniciar a respiração efetiva em sala de parto, ou seja, efetuar a transição para o ambiente extrauterino, e de reanimação propriamente dita nos neonatos pré-termo se deve, de modo geral, à sua imaturidade anatômica e fisiológica (Branco; Guinsburg, 2013). Portanto, nota-se a que necessidade de ventilação com pressão positiva e manobras avançadas de reanimação na sala de parto, é bastante frequente em prematuros, especialmente naqueles de extremo baixo peso.

Dentre os prematuros do estudo que necessitaram de atendimento em sala de parto, 42,3\% foram hospitalizados em unidade de alojamento conjunto, 32,2\% em unidade semi-intensiva neonatal e $25,5 \%$ em cuidados intensivos. A hospitalização é necessária para acompanhar e ter cuidados demasiados. No hospital estudado, a internação em unidade alojamento conjunto é de rotina para o recém-nascido e sua mãe, onde permanece durante 2 dias e recebem alta em seguida.

Aos que foram internados em unidades de cuidados intensivos e semi-intensivos, os motivos desta hospitalização variavam de disfunção respiratória $(41,2 \%)$, hipoglicemia $(1,5 \%)$, icterícia $(2,7 \%)$, infecção perinatal $(1,2 \%)$, insuficiência cardíaca $(0,9 \%)$, entre outros $(11,3 \%)$, sendo a disfunção respiratória o principal motivo das internações.

Dificuldades adaptativas que recém-nascido prematuro apresenta, destaca-se suscetibilidade aumentada ao estresse oxidativo e às infecções, o que facilita o aparecimento de morbidades que contribuem para a mortalidade neonatal desse grupo de pacientes (Almeida et al., 2010).

A disfunção respiratória apresentada na maioria dos prematuros, justifica a necessidade do $\mathrm{O}^{2}$ inalatório em sala de parto e em alguns casos da ventilação por pressão positiva. A literatura aponta que ao nascimento, o prematuro frequentemente tem uma deficiência respiratória, requerendo uma ventilação mecânica através de tubos nasotraqueais ou orotraqueais (Salge et al., 2009). Um estudo sobre fatores neonatais associados à prematuridade, aponta que dos grupos de doenças de base fetais o mais frequente, doenças do aparelho respiratório tiveram 81 casos (78\%) (Salge et al., 2009).

A respiração logo após o nascimento é pouco efetiva, uma vez que há imaturidade estrutural dos pulmões, do sistema surfactante, da musculatura e da caixa torácica, acompanhada de imaturidade do sistema nervoso central responsável pelo controle do ritmo respiratório (Branco; Guinsburg, 2013). E estudos apontam a grande ocorrência de doenças respiratórias em crianças até um ano de idade (Martins et al., 2014)

A reanimação ao nascimento é uma das oito intervenções estratégicas para diminuir a mortalidade infantil em nível mundial. Para recepcionar recém-nascidos neste período crítico, onde a chance de óbito ou morbidade é alta, é essencial contar com equipamentos apropriados e uma equipe qualificada e capacitada a realizar de forma rápida e efetiva os procedimentos avançados de reanimação, de acordo com o estado da arte no que tange aos conhecimentos existentes (Branco; Guinsburg, 2013).

\section{CONSIDERAÇÕES FINAIS (ou Conclusão)}

Este estudo proporcionou o identificar o perfil dos recém-nascidos prematuros do município de Feira de Santana, Bahia. Estes resultados apontam a necessidade de preparação de profissionais a nível de conhecimento e cuidados clínicos avançados, 
para planejamento de medidas eficazes para reduzir complicações ao nascimento e também serem capacitados, a ponto de saber lidar com a prematuridade.

Os dados deste estudo evidenciaram as consequências imediatas da prematuridade para os recém-nascidos ao revelar os escores de Apgar e baixo peso ao nascimento. Reconhecer o prognóstico das variáveis de desenvolvimento dos prematuros, pois prematuros cada vez menores e mais imaturos impõe o questionamento quanto à qualidade de sua vida futura.

O presente estudo também sugere que deve haver um aumento da atenção clínica para as disfunções respiratórias apresentadas pelos prematuros do estudo, e consequentemente o preparo do suporte necessário aos recém-nascidos. Além disso, é de extrema importância um estudo das estratégias de intervenções potenciais durante o parto prematuro, e reformulação da prática clínica das equipes em unidades obstétricas e neonatais do centro em estudo.

Com base no exposto, é importante planejar e desenvolver ações estratégicas a nível municipal, voltadas para a melhoria das condições de saúde neonato. As informações geradas são fundamentais, sugerindo que favoreça o processo de tomada de decisão de maneira estratégica, determinar prioridades e metas, somando esforços e adotando responsabilidades para abranger melhorias à assistência de prematuro.

Sugere-se o desenvolvimento de mais estudos, tendo em vista o importante papel da prematuridade relacionada a mortalidade infantil, é imprescindível que pesquisas com essa temática tenham continuidade para esclarecer as causas da prematuridade, com finalidade de auxiliar o planejamento de ações preventivas, diminuindo, portanto, a morbimortalidade infantil.

Este estudo apresentou como limitações o fato de não poder ser analisada a prevalência dos nascimentos prematuros na cidade de Feira de Santana-Bahia por trata-se de um estudo de caso-controle, e devido à ausência de alguns dados, considerados como ignorados no estudo, a pesquisa pode estar sujeita a viés de memória.

\section{REFERÊNCIAS}

ALMEIDA, M.F.; GUINSBURG, R.; COSTA, J.O.; ANCHIETA, L.M.; FREIRE, L.M.; CAMPOS, D. JR.Non-urgent caesarean delivery increases the need for ventilation at birth in term newborn infants. ArchDisChild Fetal Neonatal Ed 2010;95(5):F326-30. BRANCO, M.F.; GUINSBURG, R. Programa de Reanimação Neonatal da Sociedade Brasileira de Pediatria: Condutas. São Paulo: SBP; 2013.

BRASIL. Ministério da Saúde. O Brasil está entre os dez países com o maior número de partos prematuros, aponta OMS. 2012. Disponível em: $<$ http://www.brasil.gov.br/noticias/arquivos/2012/05/04/brasil-esta-entre-os-dez-paisescom-o-maior-numero-de-partos-prematuros-aponta-oms $>$. Acesso em: 20 de setembro de 2015.

CASCAES, A.M.; GAUCHE, H.; BARAMARCHI, F.M.; BORGES, C.M.; PERES, K.G. Prematuridade e fatores associados no Estado de Santa Catarina, Brasil, no ano de 2005: análise dos dados do Sistema de Informações sobre Nascidos Vivos. Cad. Saúde Pública, Rio de Janeiro, mai 2008.

CREMONESE, C.; FREIRE, C.; MEYER, A.; KOIFMAN, S.Exposição a agrotóxicos e eventos adversos na gravidez no Sul do Brasil, 1996-2000. Cad. Saúde Pública, Rio de Janeiro, 28(7):1263-1272, jul, 2012 
CUNHA, A.L.; FERNANDES, D.S.; MELO, P.F.; GUEDES, M.H. Fatores associados à asfixia perinatal. RevBrasGinecolObstet 2004 nov/dez; 26 (10): 799-805.

DOCUMENTAL, Brasil, no ano de 2005: análise dos dados do Sistema de Informações sobre Nascidos Vivos. Cad. Saúde Pública, Rio de Janeiro, mai 2008.

DRUMMOND, S.; SOUZA, T.S.; LIMA, F.G.; VIEIRA, A.A.Correlação entre o uso de corticoterapiaantenatal, a reanimação e a mortalidade de recém-nascidos prematuros de muito baixo peso. RevBrasGinecol Obstet. 2014; 36(5):211-5

HOWSON, C.P.; KINNEY, M.V.; LAWN, J.E. MarchofDimes, PMNCH, SavetheChildren, WHO. Born Too Soon: The Global ActionReportonPretermBirth. World Health Organization. Geneva, 2012;

MARTINS, C.B.G; BARCELON, A.A.; LIMA, F.C.A; GAÍVA, M.A.M. Perfil de morbimortalidade de recém-nascidos de risco. CogitareEnferm. 2014 Jan/Mar; 19(1):109-15

MOTTA ME, SILVA GA, ARAÚJO OC, LIRA PI, LIMA MC. O peso ao nascer influencia o estado nutricional ao final do primeiro ano de vida? J Pediatr 2005 set/out; 81(5):377- 82.

MWAMAKAMBA, L.W.; ZUCCHI, P. Estimativa de custo de permanência hospitalar para recém-nascidos prematuros de mães adolescentes em um hospital público brasileiro. einstein. 2014;12(2):223-9

OLIVEIRA, L.L.; GONÇALVES, A,C.; COSTA, J.S.D.; BONILHA, A.L.L. Fatores maternos e neonatais relacionados à prematuridade. RevEscEnferm USP . 2016;50(3):382-389

OLIVEIRA, N.C.; MOURA, E.R.F.; SILVA, C.F. Perfil dos nascidos vivos de maranguape-ce: a partir da série histórica de 2000 a 2003 Rev. RENE. Fortaleza, v. 8, n. 1, p. 52-59, jan./abr.2007

PICCOLI, A.; SOARES, C.R.S.; COSTA, G.; SILVEIRA, J.L.; FIATT, M.P.; CUNHA, R.S. Perfil clínico de neonatos de muito baixo peso internados em uma Unidade de Tratamento Intensivo Neonatal. Revista HCPA. 2012;32(4):412-419

RADES Ê, BITTAR RE, ZUBAIG M. Determinantes diretos do parto prematuro eletivo e os resultados neonatais. RevBrasGinecol Obstet. 2004;26(8):655-62.

RAMOS, H.A.C.; CUMAN, R.K.N.Fatores de risco para prematuridade: pesquisa documental. Esc Anna Nery RevEnferm 2009 abr-jun; 13 (2): 297-304

REGO, M.A.S.; FRANÇA, E.B.; TRAVASSOS, A.P.A; BARROS, F.C. Avaliação do perfil de nascimentos e óbitos em hospital de referência. Jornal de Pediatria - Vol. 86, $\mathrm{N}^{\circ} 4,2010$

SALGE, A.K.M.; VIEIRA, A.V.C.; AGUIAR, A.K.A.; LOBO, S.F.; XAVIER, R.M.; ZATTA, L.T.; CORREA, R.R.M.; SIQUEIRA, K.M.; GUIMARÃES, J.V.; ROCHA, K.M.N.; CHINEM, B.M.; SILVA, R.C.R. Fatores maternos e neonatais associados à prematuridade. Rev. Eletr. Enf. [Internet]. 2009;11(3):642-6. Disponivel em: http://www.fen.ufg.br/revista/v11/n3/v11n3a23.htm.

SILVA, G.F; PELLOSO, S.M. Perfil das parturientes e seus recém-nascidos atendidos em um hospital-escolas do Noroeste do Estado do Paraná. Rev Esc Enferm USP 2009; 43(1):95-102

STOLL, B.J.; HANSEN, N.I.; BELL, E.F.; SHANKARAN, S.; LAPTOOK, A.R.; WALSH, M.C. Neonatal outcomes of extremely preterm infants from the NICHD Neonatal Research Network. Pediatrics 2010;126:443- 56.

World Health Organization (WHO). The optimal duration of exclusive breastfeeding. Geneva: World Health Organization; 2002. Disponívelem: http:// whqlibdoc.who.int/hq/2001/WHO_NHD_01.08.pdf 